\title{
Cyberbullying: intimidación entre adolescentes a través de la red social Facebook ${ }^{1}$
}

\author{
Astrid Carolina Gómez Almanza ${ }^{2}$ \\ Universidad Pedagógica Nacional, Bogotá, Colombia ${ }^{3}$ \\ gomezastridcarolina@gmail.com \\ Diana Castillejo ${ }^{4}$ \\ Universidad Del Magdalena - Colombia \\ dianacastillejo@gmail.com \\ Germán Vargas 5 \\ Universidad Pedagógica Nacional- Bogotá, Colombia \\ gevargasqu@gmail.com
}

1 Artículo de investigación realizado como parte del programa de Maestría de Tecnologías de la Información y la Comunicación Aplicadas a la Educación. Universidad Pedagógica Nacional, Bogotá, Colombia.

2 International teacher license with speciality in linguistically diverse education. Denver, Colorado. Department of Educaction. Estados Unidos.

3 Estudiante de la Maestría de Tecnologías de la Información y la Comunicación Aplicadas a la Educación.

4 Especialista en pedagogía infantil. Docente de la Secretaría de Educación del Departamento del Magdalena. Estudiante de la Maestría de Tecnologías de la Información y la Comunicación Aplicadas a la Educación. Universidad Pedagógica Nacional, Bogotá, Colombia.

5 Licenciado en física. Docente de Física en la Secretaria de Educación Distrital, Bogotá. Estudiante de la Maestría de Tecnologías de la Información y la Comunicación Aplicadas a la Educación. Universidad Pedagógica Nacional, Bogotá, Colombia. 


\title{
Cyberbullying: intimidación entre adolescentes a través de la red social Facebook
}

\section{Resumen}

El objetivo del presente artículo fue describir de que manera un grupo de jóvenes de grado sexto pertenecientes al colegio distrital León de Greiff en la ciudad de Bogotá, se estaban viendo afectados en su convivencia escolar por el uso inadecuado de Facebook. La investigación fue de tipo cualitativo, con diseño de estudio de caso y una muestra representativa de 16 estudiantes de género femenino entre los 11 y 13 años de edad. En la recolección de datos se utilizaron entrevistas, diarios de campo/bitácoras, observación participante y sistemas de registro tecnológico. Para triangular la información, se utilizó el programa ATLAS TI, donde se analizaron las categorías en contexto, cyberbullying, información, intimidación, Facebook y sujeto. Se efectuó un proceso de codificación, categorización y nivel conceptual. Los resultados mostraron que los 16 estudiantes utiliza Facebook con fines intimidantes de forma directa, indirecta o de intimidación social /relacional.

Palabras clave: Cyberbullying, convivencia escolar, intimidación, Facebook

\section{Cyberbullying: teen bullying through Facebook social network}

\begin{abstract}
The aim of this paper was to describe how well a group of sixth-grade youth belonging to Leon de Greiff district school district from the city of Bogotá, DC, were suffering in their school life due to the inappropriate use of Facebook. The research was a qualitative one with case study design and a sample of 16 female students between 11 and 13 years old. In the data collection, interviews, field diaries / logs, participant observation and recording technology systems were used. To triangulate the information, the IT program ATLAS, where categorised according to context, cyber bullying, information, intimidation, Facebook and subject analysis were used. A process of coding, categorization and conceptual level was performed. The results showed that 16 students used Facebook with intimidating for direct, indirect or social / relational bullying manner.
\end{abstract}

Keywords: Cyber bullying, school life, bullying, Facebook

\section{intimidação entre adolescentes por meio da rede social Facebook}

\section{Resumo}

O objetivo deste artigo é descrever a maneira na qual um grupo de jovens do sexto ano, da escola pública "León de Greiff" na cidade de Bogotá D.C, estavam sendo afetados na sua convivência escolar pelo uso inadequado do Facebook. A pesquisa é de tipo qualitativo, e possui um estudo de caso e uma amostra representativa de 16 estudantes mulheres, entre os 11 e 13 anos. Na coleta de dados, foram usadas pesquisas, diários de campo, observação participante e sistemas de registro tecnológico. Para triangular a informação, usou-se o programa ATLAS TI, no qual se analisaram as categorias de contexto, cyberbullying, informação, intimidação, Facebook e sujeito. Finalmente, realizou-se um processo de codificação, categorização e nível conceitual. Os resultados mostraram que as 16 estudantes usam Facebook com fins de intimidação de forma direta, indireta ou de intimidação social/relacional.

Palavras chave: Cyberbullying, convivência escolar, intimidação, Facebook 


\section{Introducción}

Si bien, las nuevas tecnologías de la información y la comunicación, y su incorporación al nuevo milenio, han traído consigo muchísimas ventajas como el intercambio de contenidos y nuevos ambientes virtuales de aprendizaje, con el pasar del tiempo y sin darnos cuenta, también han potenciado una nueva forma de intimidación y acoso entre jóvenes conocida como Cyberbullying. De acuerdo con Pörhölä y Kinney se trata de el uso de nuevas tecnologías de la comunicación como internet, correo electrónico páginas web y redes sociales que se usan para difundir información negativa sobre individuos. Este fenómeno ha aumentado debido a que cada vez más son muchos los que utilizan las tecnologías para difamar, coaccionar, amenazar, insultar y hacer daño a los demás. Afirman Pörhölä y Kinney que "[...] con la llegada de las nuevas tecnologías de la comunicación los humanos tienden a encontrar formas ingeniosas de usarlas para herir y perjudicar a otros [...]"(Pörhölä y Kinney, 2010). Este es el caso de la joven Amanda Tood, un caso de Cyberbullying reportado por la Política Nacional de Uso Responsable de las TIC del Ministerio de Tecnologías de la Información y las Comunicaciones de la república de Colombia. (www.enticconfio.gov.co), en donde se relata como una joven canadiense de 15 años fue hallada muerta luego de haber sufrido sextorsion, ${ }^{6}$ a través de la red social Facebook, por un intercambio de material de contenido intimo entre sus amigos que ella había hecho de manera irresponsable. La joven no aguanto tantas burlas, presión, intimidaciones y amenazas en Facebook, era casi como una pesadilla, por lo cual decidió quitarse la vida .

Es importante que las instituciones educativas del siglo XXI conozcan este y muchos casos de ciberacososo, que han venido ocurriendo en todo el mundo, de modo que se eduque a los alumnos y futuros profesionales en el uso adecuado de las nuevas tecnologías de la información y la comunicación para el fortalecimiento de su propio desarrollo psicológico, emocional, social y físico.

La presente investigación mostrará la experiencia de un estudio de caso llevado a cabo con 16 jóvenes de grado sexto del colegio distrital León de Greiff en el barrio lucero bajo, localidad Ciudad Bolívar en Bogotá, Colombia. Primero presentaremos un grupo de fundamentos teóricos entorno a nuestra temática cyberbullying, luego de ello abordaremos el diseño, metodológico utilizado y finalmente se explicaran los resultados y Conclusiones principales del estudio.

6 La Sextorsión o sexting es una forma de explotación sexual, que está en acelerado aumento en internet por la proliferación de webs de contactos y chats para ligar. Tomado de: http://www.protestantedigital. com/ES/Sociedad/articulo/11901/El-chantaje-de-la-sextorsion-crece-en-internet 


\section{Fundamentos Teóricos}

\section{Ciberbullying, forma virtual de intimidación escolar.}

Con el pasar del tiempo, y el enorme crecimiento tecnológico, una innumerable cantidad de escenarios empiezan a tomar caminos inesperados, tal es el caso de los espacios cotidianos de socialización, los cuales en la actualidad trascienden la presencialidad, permitiendo romper posibles barreras como la distancia y el tiempo, todo esto como consecuencia de la facilidad de conectarse con otros a través de un medio virtual. En este medio tan particular, se crean las denominadas redes sociales (Imaña, 2008), en donde la población encontró un lugar para estar "conectado" y obtener beneficios. en una cultura donde la inmediatez es algo necesario e inminente, y en donde grupos comunes se sienten identificados de manera permanente, generando diferente cantidad de reacciones inusitadas y procesos masivos, que hasta la fecha no habían sido posibles, de no ser por las particularidades que este tipo de conglomerados y su respectiva forma de conexión, aporta a lo que se tenía entendido como socialización (Blandir y Nava, 2011). Estos nuevos espacios de contacto social, dan lugar a que se repliquen ciertos patrones y conductas cotidianas, como es el caso de los conflictos y su manejo, surge ahora como motivo de alarmante preocupación, dada la capacidad del medio virtual y de los alcances que pueda tener al ser usado de forma inadecuada. En este sentido, una problemática que ha surgido, y que viene proliferando entre los escenarios escolares, es el denominado ciberbullying, entendido como "[...] agresiones intencionales, continuas, que el o los estudiantes, con mayor poder dado por su fuerza física, su popularidad o su capacidad para manejar y controlar a sus pares, ejercen sobre otros considerados incapaces de defenderse, con la intención de generar malestar, temor o daño y, así mismo, con el propósito de incrementar (los intimidadores) su condición de dominación [...]" (Cuevas, Hoyos, y Ortiz, 2009 en Noruega, Olweus, 1973).

Esta problemática en particular, permea constantemente los espacios educativos, dada la gran facilidad de acceso y uso de las nuevas tecnologías por parte de los estudiantes, agravado también por el hecho, de brindar espacios que no presentan ninguna supervisión o regulación, lo que facilita la "libre expresión" de sus usuarios y el poco control por parte de adultos como docentes y padres. Desde esta óptica educativa, está claro como estos procesos de acoso no vienen dados sólo en ciertas zonas en el mundo, sino que permean a muchos países (Ghiso y Yanet, 2010), (Lopez, 2008), (Galvis, 2011), (García-Maldonado, Joffre-Velázquez, Martínez-Salazar, y Llanes-Castillo, 2011), (Sonja, Julian, ShawThérèse, y Cross., 2010), (Smith, Jess, Manuel, Sonja, Russell, y Neil, 2008). De tal forma, que la intimidación y diversas formas de agresión que se vienen presentando, reiteran la importancia de implementar un manejo acertado de espacios virtuales, monitoreo y supervisión de personal o legislaciones responsables y una constante preocupación investigativa por consolidar un marco referencial sólido, que ayude a mermar los efectos devastadores de esta situación. 
Es así, que el uso de las tecnologías ha diversificado las posibles formas de intimidación conocidas, y y de esta manera ha ampliado la magnitud de su impacto y la diversidad de sus formas. De esto no hablan (Smith, et al., 2008) quienes enumeran algunos spectos particulares, que distinguen el ciberacoso del acoso tradicional:

- No hay un sitio donde esconderse.

- La audiencia es más amplia.

- Hay una invisibilidad de los acosadores.

Los usuarios escolares se incluyen en este grupo, y con el uso constante del ciberespacio pueden desarrollar un mayor manejo de estas herramientas tecnológicas, incrementando a su vez, las formas de acoso que no se contemplaban anteriormente. Un ejemplo claro de la transformación del acoso por el uso del internet, lo plantean (García-Maldonado, et. al, 2011) en la tabla 1, en donde de manera compartiva, dan una visión más amplia de la situación actual del acoso escolar:

Tabla 1. Diferencias entre bullying y ciberbullying.

Bullying

Cara a cara

En grupo o individual

Golpes, empujones, agresión verbal o exclusión social

Sólo en horas de escuela

Se limita a la agresión directa

Sólo audiencia escolar

Certeza de que sólo se encuentra en el ámbito escolar

\section{Ciberbullying}

Anónima

Individualmente

Mensajes, e-mail, imágenes manipuladas

En todos lados y a toda hora

Sin límite de alcance

Audiencia mundial por Internet

Incerticumbre de no saber quién ha visto las imágenes o mensajes

Tomado de (García-Maldonado et al., 2011)

De esta manera podemos observar como situaciones cotidianas escolares, ahora trasladadas a escenarios virtuales, han amplificado y diversificado las formas de efectividad de acoso por medios electrónicos, el cual se fortalece en la medida en que éstos brinden las posibilidades para su proliferación y logre consolidarse en espacios tan populares y de uso cotidiano como la red social Facebook. Las herramientas dadas por la tecnología que en un principio cumplieron su cometido mediático, presentan en escenarios escolares una cara de la moneda no tan agradable, en la que muestran como las posibilidades que ahora obtienen los usuario, los acorazan de una serie de beneficios para reincidir y ahondar posibles problemáticas de socialización presentadas entre pares escolares. 
Podemos entrever como el acoso continua siendo acoso, indistintivamente del escenario en que se dé (virtual o presencial) o de la herramienta que se utilice para cumplir con su cometido, hacerle daño al otro. Lo más importante es lograr dimensionar las particulares de cada escenario y las nuevas connotaciones, debido a la innegable afectación que tiene en los procesos y dinámicas sociales de los espacios escolares en la actualidad.

\section{Diseño metodológico}

Para cumplir con los propósitos expuestos en esta investigación se consideró pertinente adoptar la metodología de estudio de caso, enmarcada en el paradigma cualitativo, debido a que este examina o indaga sobre un fenómeno en su entorno real a partir de una reflexión que parte de los procesos que en esta se desarrollan, dentro de este marco se utilizará entonces la observación participante, la cual de acuerdo a (Burgos, 2003) implica de manera necesaria, una intensa y sostenida interacción entre el investigador y las personas —objeto de estudio- dentro de su propio contexto. Durante el periodo de realización, el investigador recoge la información que exigen sus objetivos de búsqueda. Así mismo, observar sus conflictos problemáticas que le permitan ir visualizando los elementos que van emergiendo.

En concordancia con esto Merriam define que "Un estudio de caso cualitativo es una descripción y análisis exhaustivo y holístico de una instancia individual, de un fenómeno o de una unidad social" (Merriam, 1998, p.9). De esta forma, esta postura atiende a las necesidades de nuestra investigación, ya que el principal objetivo de ésta es estudiar si el uso inadecuado de Facebook afectaba la convivencia escolar entre los estudiantes del grado 601 del Colegio Distrital León de Greiff .

\section{¿Dónde?}

Este estudio se llevo a cabo con un grupo de 16 estudiantes de grado sexto del Colegio Distrital León de Greiff ubicado en el barrio Lucero Bajo en la localidad de Ciudad Bolívar en Bogotá, Colombia. El Colegio Distrital Liceo León de Greiff, es una institución de educación básica-media, calendario A, de carácter mixto, que ofrece los niveles de educación básica y media en tres jornadas mañana, tarde y noche. El colegio tiene 31 años de servicio a la comunidad, y cuenta con una población de 700 estudiantes, los cuales en su mayoría pertenecen al estrato uno y dos, y algunos de ellos provienen de población desplazada o reinsertada.

\section{¿Con Quiénes?}

Las personas que participaron en este estudio de caso fueron 16 estudiantes con edades entre los 11 y 13 años de edad, de género femenino, que cursan actualmente grado sexto en el Colegio Distrital León de Greiff en la jornada de la tarde, en el 
barrio Lucero Bajo, localidad Ciudad Bolívar en Bogotá, Colombia. Cada uno de los alumnos descritos en esta investigación, tienen previo consentimiento de sus padres o acudientes. Existe un asentimiento informado impreso y firmado por cada uno de los acudientes.

\section{¿Con qué instrumentos?}

como mecanismo de recolección de datos, se utilizaron la entrevista semiestructurada, y los diarios de campo o bitácoras para la observación de cada participante. De manera adicional, se utilizaron técnicas de registro complementario como son los sistemas de registro tecnológico a través de la recolección de material de audio y fotografías para soportar la investigación.

Diarios de campo o bitácoras: De acuerdo (Villegas Ramos, 2011), la bitácora o diario de campo se caracteriza por ser un instrumento que permite recoger información sobre la problemática planteada en una investigación. Ha sido utilizado por los antropólogos e investigadores como cuaderno de notas, en donde apuntaban sus observaciones sobre la problemática que estaban investigando. En nuestro estudio de caso, las bitácoras fueron utilizadas para consignar información concreta, proveniente de la observación del participante y en donde se describía el comportamiento de cada categoría establecida en nuestra investigación, de modo que en cada momento el investigador estuviera enfocado en recopilar la mayor cantidad de datos, enriqueciendo y dotando de rigurosidad al proceso investigativo.

Entrevista Semi-Estructurada: Según Baez y col. la entrevista semi estructurada es aquella en la que se trabaja con unos contenidos y un orden preestablecido, dejando abierta la gama de posiciones que el entrevistado desee manifestar, y se utiliza predominantemente para conocer la imagen (elementos, rasgos, cualidades, actitudes y motivaciones). En nuestro estudio de caso, una vez completado el proceso de observación participante, se procedió a realizar las entrevistas con una muestra intencional de las niñas involucradas en el conflicto, para obtener datos valiosos por parte de los mismos actores involucrados (Báez y Pérez de Tudela, 2009).

Sistemas de registro tecnológico: De manera adicional a las entrevistas y observaciones, se contó con un registro complementario, a través de la recolección de material de audio y de imágenes (pantallazos Facebook) según las particularidades del ambiente observado.

\section{El proceso de triangulación}

en una investigación cualitativa los datos pueden provenir de diferentes fuentes y el análisis de los mismos constituye una tarea compleja para el investigador, por eso para facilitar la interpretación de la información recolectada en nuestro estudio, 
utilizamos un programa del tipo CAQDAS (Software para el análisis de datos cualitativos asistidos por ordenador), denominado ATLAS TI, como una herramienta que nos permitiera lograr una mayor optimización de los datos, así como una codificación y categorización clara, para luego proceder a analizar los resultados obtenidos. El siguiente orden representa el proceso que seguimos en ATLAS TI para analizar todos los datos e información recolectada:

- Codificación

- Categorización

- Nivel conceptual

- Gráfico (Análisis, Conclusiones y resultados)

Para (Taylor \& Bogman, 1986) en la investigación cualitativa, la codificación es un modo sistemático de desarrollar y refinar las interpretaciones de la información recolectada, el proceso de codificación incluye la reunión y análisis de todos los datos que se refieren a temas, ideas, conceptos, interpretaciones y proposiciones. El primer paso para triangular fue crear un proyecto en el programa ATLAS TI y asignarle un nombre al mismo. Luego de eso en el icono de documentos primarios o P docs se procedió a cargar todos los documentos considerados pertinentes en la investigación: antecedentes, bitácoras de la observación participante que se hizo en Facebook durante todo el proceso investigativo, también se adicionó una tabla de categorías con sus respectivos autores, y el resumen de las entrevistas y grupos focales que se realizaron a los estudiantes, para tener un consolidado de toda la información.

Cada archivo se organizó consecutivamente de la siguiente manera, P1, P2, P3, etc. y luego, para codificar cada documento, se inició con los antecedentes y de acuerdo a la tabla que se tenía consolidada, se creaba un code name específicamente para los párrafos y se asociaba con la lista de de categorías de autores previamente establecidos. El mismo procedimiento se realizó para las bitácoras, en donde, no sólo se asignaban codes para la información en (texto) allí contenida, sino también para las fotografías anexadas a las mismas bitácoras. Luego se procedió a hacer una lista de memos, los cuales se escribían con títulos muy precisos y con una descripción muy detallada, aquí también se inicia el proceso de categorización, esto si vemos que, el propósito de los memos era asociarlos y clasificarlos con los codes previamente establecidos en los documentos e ir de esta manera, generando un orden en toda la información.

Según (Taylor \& Bogman, 1986), el número de categorías que se adopten dependerá de la cantidad de datos recogidos y de la complejidad de nuestro esquema analítico. Para Tesh, en la teoría fundamentada "Algunas categorías pueden ser derivadas de teorías existentes, pero este procedimiento es flexible" (Tesh, 1990, 
p. 85), vemos que en esta fase es donde empiezan a emerger los datos y a generarse el agrupamiento de los codes o códigos, mostrando las relaciones entre éstos y su incidencia en las distintas categorías expuestas.

En cuanto al nivel conceptual se relacionaron los vínculos con los diferentes códigos y categorías, observando de este modo, los elementos centrales en los datos, que permiten ser vistos a través de representaciones visuales, que muestran el todo de forma coherente. Al aplicar el software Atlas ti, éstas relaciones se muestran a través de los elementos denominados Networks, en nuestro trabajo creamos una familia principal con el nombre de "Uso inadecuado de Facebook "y debajo de eso creamos dos codes estableciendo dos hipótesis, la primera "No afecta la convivencia escolar de manera negativa" y el otro "Afecta la convivencia escolar de manera negativa". Lo que hicimos fue establecer un paralelo entre esas dos hipótesis y arrastrar las diferentes categorías con las preguntas y respuestas de la entrevista semi-estructurada y grupos focales que se le aplicó a los estudiantes, cada pregunta esta referenciada bajo un número, el cual representa el Id o identificación del documento, cuando fue cargado en la sección P-docs del programa. Luego de eso, el software Atlas ti conectaba automáticamente las categorías con las respectivas preguntas y respuestas y se asociaban con cada una de ellas, tal como lo muestra la figura 1.

FIGURA 1. Paralelo -Uso inadecuado de Facebook

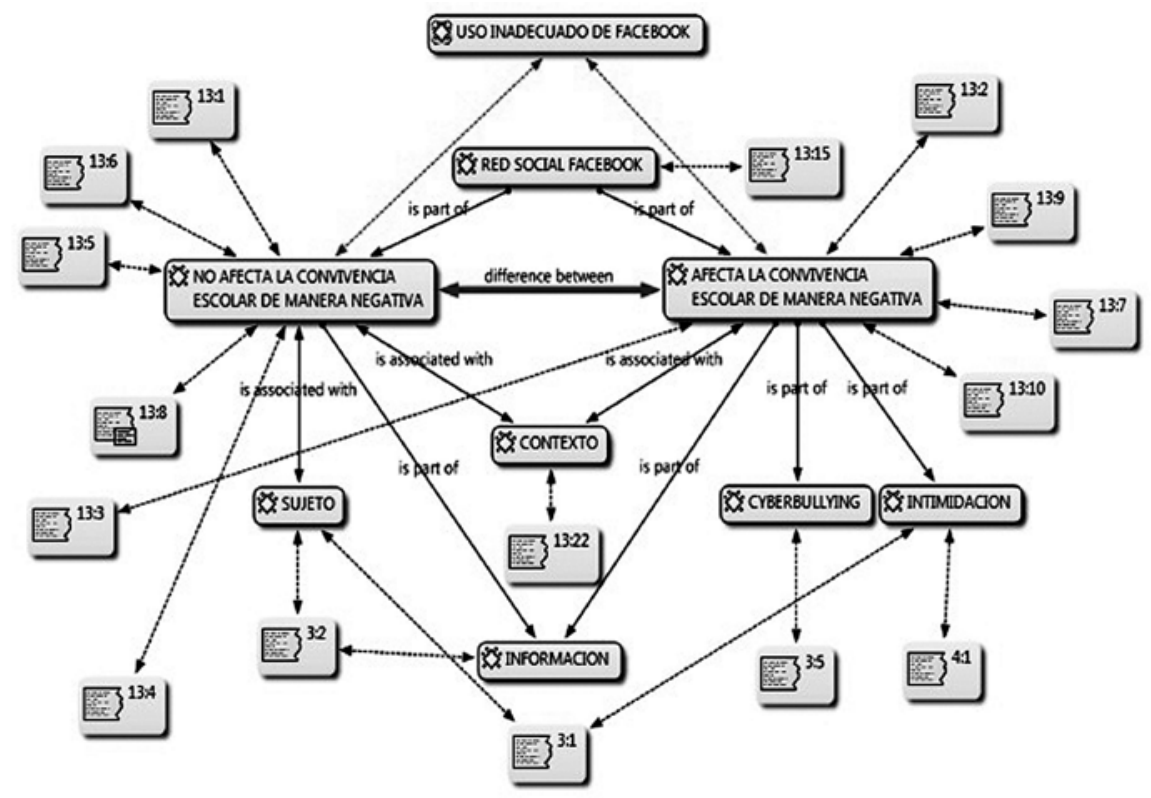

Realizado en ATLAS TI 
Para el proceso de triangulación realizamos un segundo gráfico, el cual asociaba y conectaba las diversas categorías previamente establecidas en nuestro estudio: contexto, cyberbullying, información, intimidación, Facebook y sujeto. Luego cada una de las categorías fue asociada con las bitácoras de observación participante y con una lista de memos que fueron creados y que representaban las sub-categorías en nuestro estudio. Este segundo grafico también nos daba una segunda mirada y nos ayudaba a conectar toda la información y documentos que teníamos para nuestra investigación.

FIGURA 2. Categorías-

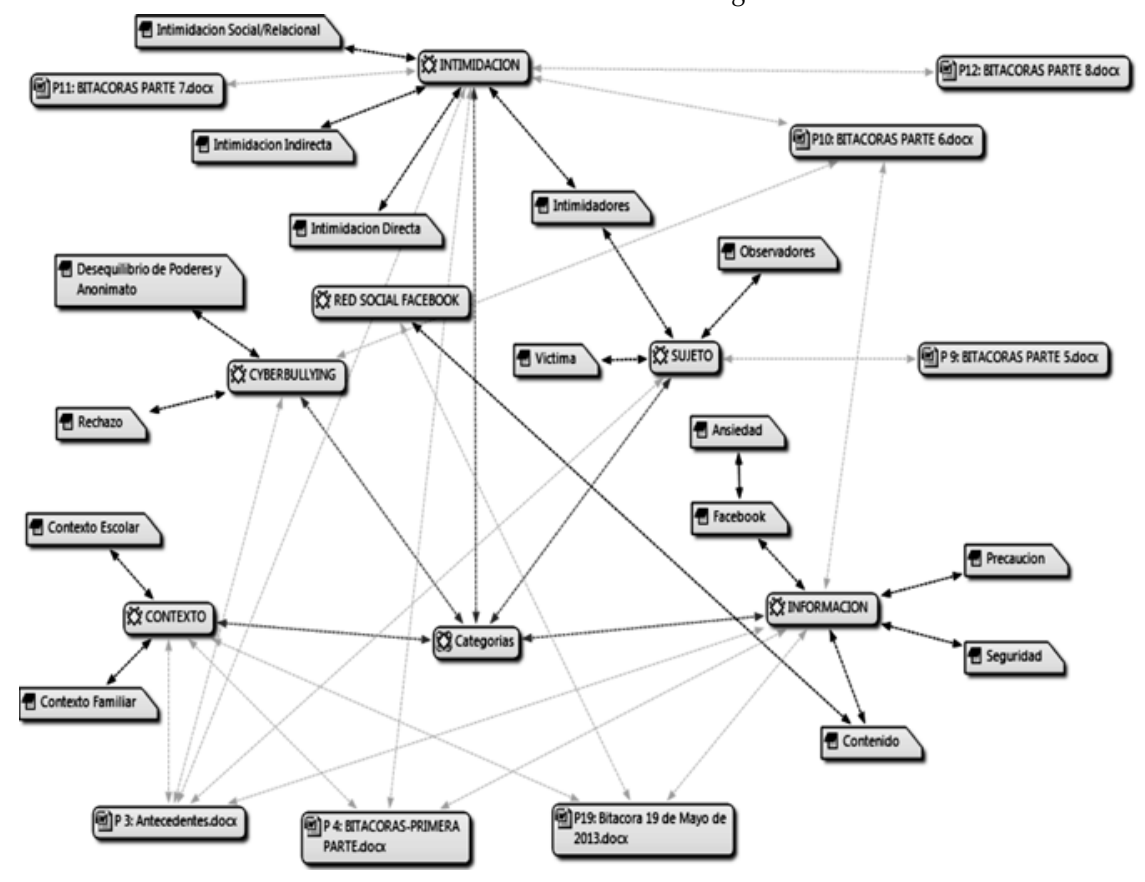

realizado en ATLAS TI 
Para darle respuesta a nuestra pregunta de investigación, sobre si el uso inadecuado de Facebook afecta la convivencia escolar entre los estudiantes del grado 602 del colegio León de Greiif, realizamos un análisis de concurrencia en las categorías, con el fin de determinar cuál de todas de las que teníamos establecidas, era la de mayor incidencia y la que se presentaba con mayor frecuencia entre los estudiantes.

Para ello lo primero que hicimos, fue dirigirnos a la barra de menú del programa Atlas ti y seleccionar el icono Analysis-Codes-Primary documents-Table, en donde seleccionamos los codes cyberbullying, información, intimidación, red social, sujeto, contexto así como los primary documents que fueron cargados en el programa, tales como: bitácoras, antecedentes y tabla de categorización y le dimos click en generar reporte. El reporte se generó de manera automática e indicó que los 16 estudiantes del grado 602 del colegio León de Greiff, utiliza Facebook con fines intimidantes, ya sea de manera directa, indirecta o de intimidación social / relacional, tal como se aprecia en la tabla 2.

\section{CODES-PRIMARY-DOCUMENTS-TABLE}

Report created by Super - 20/05/2013 10:03:24 p.m.

HU: [C:\Users\Carito\Desktop \METODOLOGÍA\ATLAS TI TRIANGULACIÓN.hpr7]

Code-Filter: All [8]

PD-Filter: All [10]

Quotation-Filter: All [67]

Tabla 2. Reporte-ATLAS TI

\begin{tabular}{|c|c|c|c|c|c|c|c|}
\hline & $\begin{array}{c}\text { P4: BITÁCORAS- } \\
\text { PRIMERA PARTE. } \\
\text { docx }\end{array}$ & $\begin{array}{l}\text { P9: BITÁCORAS } \\
\text { PARTE 5.docx }\end{array}$ & $\begin{array}{l}\text { P10: BITÁCORAS } \\
\text { PARTE } 6 . \text { docx }\end{array}$ & $\begin{array}{l}\text { P11:BITÁCORAS } \\
\text { PARTE 7.docx }\end{array}$ & $\begin{array}{l}\text { P12: BITÁCORAS } \\
\text { PARTE 8.docx }\end{array}$ & $\begin{array}{l}\text { P19: Bitácora } 19 \\
\text { de Mayo de } 2013 . \\
\text { docx }\end{array}$ & TOTALS: \\
\hline CONTEXTO & 3 & 0 & 0 & 0 & 0 & 2 & 5 \\
\hline CYBERBULLYING & 0 & 0 & 3 & 0 & 0 & 0 & 3 \\
\hline INFORMACIÓN & 4 & 0 & 2 & 0 & 0 & 2 & 8 \\
\hline INTIMIDACIÓN & 8 & 0 & 5 & 1 & 3 & 0 & 17 \\
\hline $\begin{array}{l}\text { RED SOCIAL } \\
\text { FACEBOOK }\end{array}$ & 0 & 0 & 0 & 0 & 0 & 3 & 3 \\
\hline SUJETO & 0 & 3 & 0 & 0 & 0 & 0 & 3 \\
\hline TOTALES: & 15 & 3 & 10 & 1 & 3 & 7 & 39 \\
\hline
\end{tabular}




\section{Conclusiones y resultados}

Es claro que los 16 estudiantes del grado 602 del colegio León de Greiff, utiliza Facebook con fines intimidantes, ya sea de manera directa, indirecta o de intimidación social/relacional entre sus pares. Estos resultados indican que se necesita más compromiso y apoyo por parte del núcleo familiar y del cuerpo docente de la institución, a la vez que se hace necesario implementar un programa que permita educar a estas estudiantes sobre el uso apropiado de las redes sociales, específicamente de Facebook, ya que el problema de intimidación, está afectando su desarrollo social y afectivo entre sus pares y dentro de su propio contexto escolar. En Colombia, recientemente se decreto la ley 1620 sobre la convivencia escolar emitida por el presidente de Colombia Juan Manuel Santos (Santos J., 2013), en la cual se inauguro el Sistema Nacional de Convivencia Escolar y Formación para el Ejercicio de los Derechos Humanos, Sexuales y Reproductivos y la Prevención y Mitigación de la Violencia Escolar. El objetivo de esta ley, en concordancia con el mandato constitucional y la Ley General de Educación, Ley 115 de 1994, es promover y fortalecer la formación ciudadana y el ejercicio de los derechos humanos, sexuales y reproductivos de los estudiantes de los niveles educativos de preescolar, básica y media, dentro y fuera de la escuela, para llegar contribuir a la formación de ciudadanos activos que aporten a la construcción de una sociedad democrática, participativa, pluralista e intercultural.

Con esta ley el Gobierno logra crear mecanismos de prevención, protección, detención temprana y de denuncia, de aquellas conductas que atentan contra la convivencia, como lo son, la violencia, la deserción escolar, el embarazo en la adolescencia, entre otros. Vemos que esta ley sancionada por el presidente de Colombia Juan Manuel Santos es una más de las muchas estrategias, sobre los diversos casos de ciberacoso presentados no solo en nuestro país sino en todo el mundo. Es importante que los jóvenes sean conscientes de su seguridad y acerca del uso apropiado de las tecnologías para su buen desarrollo psicológico y emocional. Por esta razón, este estudio de caso supone un significativo avance en el conocimiento de cyberbullying, y sugiere la necesidad de ahondar en su estudio para prevenir este tipo de violencia en las nuevas generaciones. 


\section{Referencias}

Álvarez, D., Núñez, J.C., Álvarez, L.D., González, A., Rodríguez, C., et al. (2010). Violencia a través de las tecnologías de la información y la comunicación en estudiantes de secundaria. Anales de Psicologia.

Arroyave, P. (2012 de enero-junio). Factores de vulnerabilidad y riesgo asociados al bullying. Revista CES Psicología, 5 (1), 116-125.

Báez y Pérez de Tudela, J. (2009). La investigación Cualitativa (2a Ed.). Madrid: ESIC Editorial.

Blandir, A., Nava, A. (2011). Las redes sociales: concepción, bondades y limitaciones. Formación Gerencial. 10(2).

Burgos, J. J. (2003). Investigar desde la escuela. En la investigación en la cultura escolar. Bogotá: Ed. Cooperativa Editorial Magisterio .

Castells, M. (2000). Internet y la sociedad red. Conferencia de presentación del Programa de Doctorado sobre la Sociedad de la información y el Conocimiento. Barcelona: Universitat Oberta de Catalunya.

Cuevas, M. Hoyos, P., y Ortiz, Y. (2009). Prevalencia de intimidación en dos instituciones educativas del departamento del Valle del Cauca. Pensamiento Psicológico. 6(13), 153 172.

Di Próspero, C. (2011, agosto-noviembre). Autopresentación en Facebook: un yo para el público. Revista Latinoamericana de Estudios sobre Cuerpos, Emociones y Sociedad, 4453.

Díaz, V. (2011). Mitos y Realidades de las Redes Sociales. Prisma Social. (6), 1-26.

Galvis, R. (2011). NO al Cyberbullying: Diseño, implementación y evaluación de una propuesta pedagógica de prevención integrada al área de tecnología e informática,. "Tesis de maestría no publicada", Universidad de Los Andes, Bogotá, Colombia.

García-Maldonado, G., Joffre-Velázquez, V., Martínez-Salazar, G., y Llanes-Castillo, A. (2011). Cyberbullying: Forma Virtual de Intimidacion Escolar. Rev. Colomb. Psiquiat., (40) 1.

Ghiso, A. M. y Yanet, O. O. (2010, enero - junio). Naturalización de la intimidación entre escolares: un modo de construir lo social. Revista Latinoamericana de Ciencias Sociales, Niñez y Juventud, 8(1), 535-556.

Imaña, T. (2008, mayo-junio). Facebook, Tejiendo la Telaraña de las Redes Sociales. Red de revistas científicas de América Latina, el Caribe, España y Portugal. Número Especial 1.

Lopez, L. (2008). El Cyberbullying en estudiantes del nivel medio superior en México. Documento presentado en X Congreso Nacional De Investigación Educativa. México: Área 17 convivencia, disciplina y violencia en las escuelas.

Luna, I. C. (2009). Guía Legal Sobre Ciberbullying y Grooming. INTECO-Instituto Nacional de Tecnologías de la Comunicacion- Gobierno de España.

Merriam, S. (1998). Case Study Research. USA: Library of Congress.

Peña, B. (2011). El alcance de la educación multimedia: las redes y la transformación social. Recuperado de http://www.ugr.es/ sevimeco/revistaeticanet/index.htm

Pérez, J., Bringué, X., y González, D. (2009). Cyberbullying: un análisis comparativo en estudiantes de Argentina, Brasil, Chile, Colombia, México, Perú y Venezuela. Generación 
digital: oportunitats i riscos delspúblics. La transformaciódels usos comunicatius, V Congrés Internacional Comunicació i Realitat .

Pörhölä, M., \& Kinney, T. (2010). El Acoso: Contextos, Consecuencias y Control. Madrid: Editorial Aresta.

Santamaria, F. (2008). Posibilidades pedagógicas. Redes sociales y comunidades educativas. TELOS Cuadernos de Comunicaicion e Innovacion. Recuperado de http://telos. fundaciontelefonica.com/telos/articulocuaderno.asp@idarticulo=7\&rev=76.htm

Santos, J. (2013). Ley 1620 sobre la convivencia escolar. Bogotá.

Santos, J. M. (2013). LEY 1620 Sobre la Convivencia Escolar. Sistema Nacional de Convivencia Escolar y Formación para el Ejercicio de los Derechos Humanos, Sexuales y Reproductivos y la Prevención y Mitigación de la Violencia Escolar. Bogotá, Colombia.

Smith, P. K., Jess, M., Manuel, C., Sonja, F., Russell, S., et al. (2008). Journal of Child Psychology and Psychiatry. 49(4), 376-385 .

Sonja, P., Julian, D., ShawThérèse, y Cross., D. (2010). Bullying in school and cyberspace: Associations with depressive Symptoms in Swiss and Australiana Adolescents. Child and AdolescentPsychiatry and Mental Health 2010. Recuperado de http://www.capmh.com/ content/4/1/28 .

Taylor, S., y Bogman, R. (1986). Introducción a los métodos cualitativos de investigación. Buenos Aires: Paidós. Buenos Aires: Paidós.

Tesh, R. (1990). Qualitative Research: Analysis and Software Tools. Bristol: The Falmer Press.

Velasco, C. E. (2010, 10 de febrero). Víctimas del cyberbullying, niños y adolescentes "podrían suicidarse". Periodico La Jornada. Sección: Sociedad y justicia.

Villegas, E. L. (2011). Investigacion y Practica En la Educacion de Personas Adultas. Valencia: Nau Llibre.

Enticconfio. Recuperado de http://www.enticconfio.gov.co/index.php/riesgos-jovenes/ item/312-el-caso-de-amanda-todd.html

Recibido: 9 junio 2012

Aceptado: 3 septiembre 2012

Como citar este artículo:

Gómez, A.C., Castillejo, D., Vargas, G. (2013)"

Cyberbullying: intimidación entre adolescentes a través de la red social Facebook".

Praxis Pedagógica 14, 31-44 